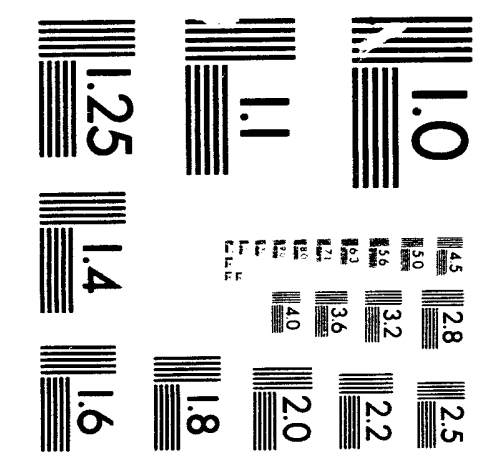



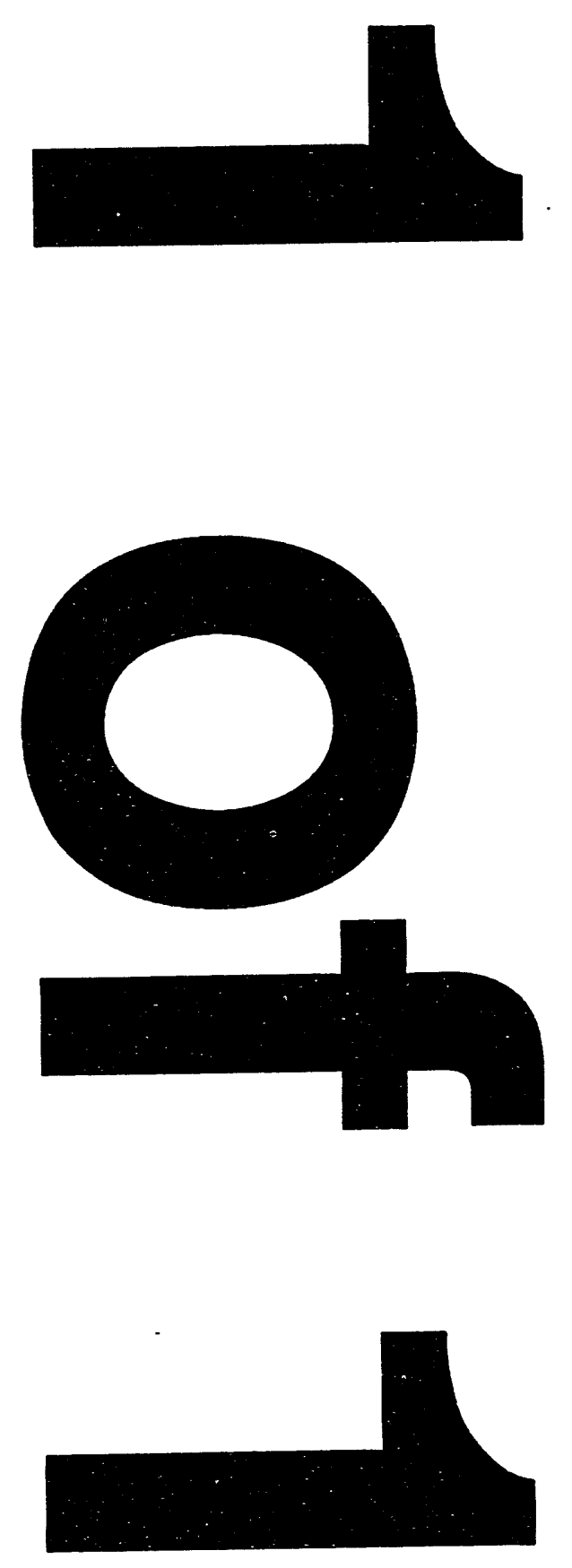
GA-A21445

\title{
UPGRADE OF DIII-D TOROIDAL MAGNETIC FIELD POWER SUPPLY CONTROLS
}

\author{
by \\ P.M. PETRACH, A.R. ROULEAU, R.D. McNULTY, \\ D.B. PATRICK, J.L. WALIN
}

This is a preprint of a paper to be presented at the 15th IEEE/NPSS Symposium on Fusion Engineering, October 11-15, 1993, in Hyannis, Massachusetts, and to be printed in the Proceedings.

\author{
Work supported by \\ U.S. Department of Energy \\ Contract DE-AC03-89ER51114
}

GENERAL ATOMICS PROJECT 3466
NOVEMBER 1993

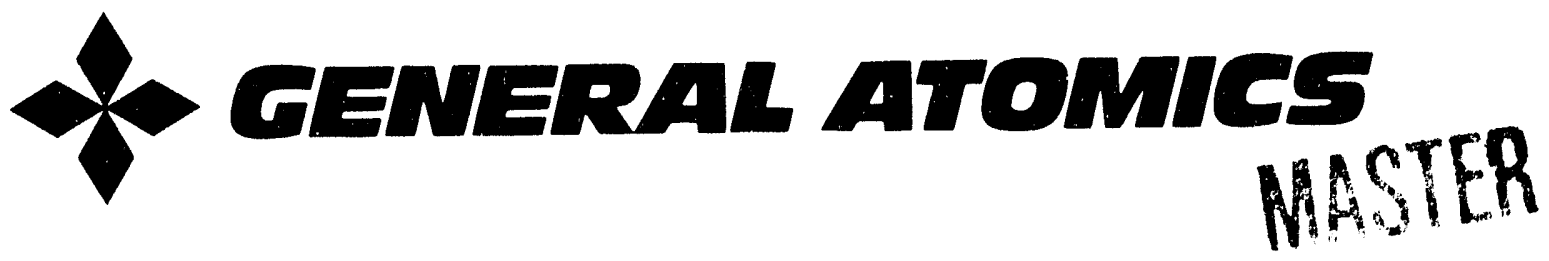
DISTRIBUTION OF THIS DOCUMENT IS UNLIMITED 


\title{
UPGRADE OF DIII-D TOROIDAL MAGNETIC FIELD POWER SUPPLY CONTROLS*
}

\author{
P.M. Petrach, A.R. Rouleau, R.D. McNulty, D.B. Patrick, J.L. Walin \\ General Atomics \\ P.O. Box 85608, San Diego, California 92138-9784
}

\begin{abstract}
The toroidal magnetic field power supply for the DIII-D tokamak is of the 12 pulse line commutated variety. It consists of four individual modules and a main system control cabinet which are combined to deliver $127,000 \mathrm{~A}$ and $1000 \mathrm{~V}$ to the toroidal field (TF) coil. The modules are connected in a series-parallel configuration but can be run alone or two at a time as well. Normally on DIII-D experiments, the series-parallel configuration is required. The original design provided each individual module with its own voltage (E) and current (I) control loop and a main control loop. A problem with this design was that the individual control loops would cause a current sharing imbalance in the parallel modules if the calibrated loops drifted by the slightest amount. It was determined that individual control loops were not needed and a single phase lock firing circuit was employed in the system cabinet with fiber optic links to the modules for gate drive signals. Since all four modules have to be on line for DIII-D to operate, a problem in any of the five E\&I control loops resulted in the supply, and, therefore, the tokamak, being idled. By reducing the number of control loops to one, the sharing problem was eliminated, as well as 4 out of 5 potential control failures. The original supply employed relay logic for sequence control and fault monitoring. There were over 130 relays in each module plus an additional 100 in the system cabinet. The combination of the number of relays with the required interconnecting wiring, the age of the supply, the virations of the cabinets and the harsh environment, resulted in a continuously escalating number of "phantom," and often intermittent, faults. In some cases, a day of testing and searching was "equired to isolate the problem. The fault and sequence logic relays were replaced by a new Programmable Logic Controller (PLC). All existing interconnect wire was removed and replaced with multiconductor cables that connect directly from fault sensors and input devices to the PLC. It $i$ anticipated that an average of $12 \mathrm{~h}$ per year in control loop downtint will be eliminated. In addition, 100 man-hours of annual calibratior; time will be eliminated. New capabilities introduced by the PLC: should provide for at least one additional plasma shot per day.
\end{abstract}

\section{INTRODUCTION}

The DIII-D tokanak fusion research program is carried out by General Atomics (GA) under contract to the U.S. Department of Energy. A Toroidal Field (TF) Power Supply is the source of power to the toroidal field coil. The TF coil consists of 144 turns of conductors surrounding the vacuum vessel and creates a toroidal field to confine the plasma. The electrical resistance and inductance of the TF coil are 4.1 milliohm and $30.6 \mathrm{mH}$ respectively. The TF power supply was originally rated to deliver $127,000 \mathrm{~A}$ of dc current to the

*Manuscript received October 14, 1993. This work was supported by the U.S. Department of Energy Contract No. DE-AC03-89ER51114.
TF coil for a time duration of $7 \mathrm{~s}$ with $10 \mathrm{~min}$ of cooling between plasma shots. The power supply was built in the 1970s using the available technology and components from that period, including relay logic for fault monitoring, timing, and sequencing. Time and environmental conditions gradually took their toll on the control boards and relays that were used for the critical control of this supply. In spite of increased maintenance, sporadic control failures became too frequent and it was evident that a major overhaul of the control system for this equipment was required.

\section{CONFIGURATION}

The TF supply consists of four individual modules that are configured in a series-parallel combination with a single system cabinet integrating all control functions (Fig. 1). The paralleled modules are referred to as PS\#1 (Mod I and II) and PS\#2 (Mod III and IV). The free-wheeling diodes that appear across the output of PS\#1 and PS\#2 provide a path for the decaying current in the toroidal field upon completion of the plasma shot. These diodes also remove the additional heat burden that the power supp!y semiconductors would bear if they had to remain in the circuit to discharge the energy in the coil. In adcition, the diofes complete the circuit when only one of the rownew supplies is operating. The original design allowed each one of these modules to be used as an independent power supply. Each module had its own controls and lise ability 10 run rogether with the other modules by selectici from the main system controller. The individual modkit ons frequenty thad to be calibrated to be identical to

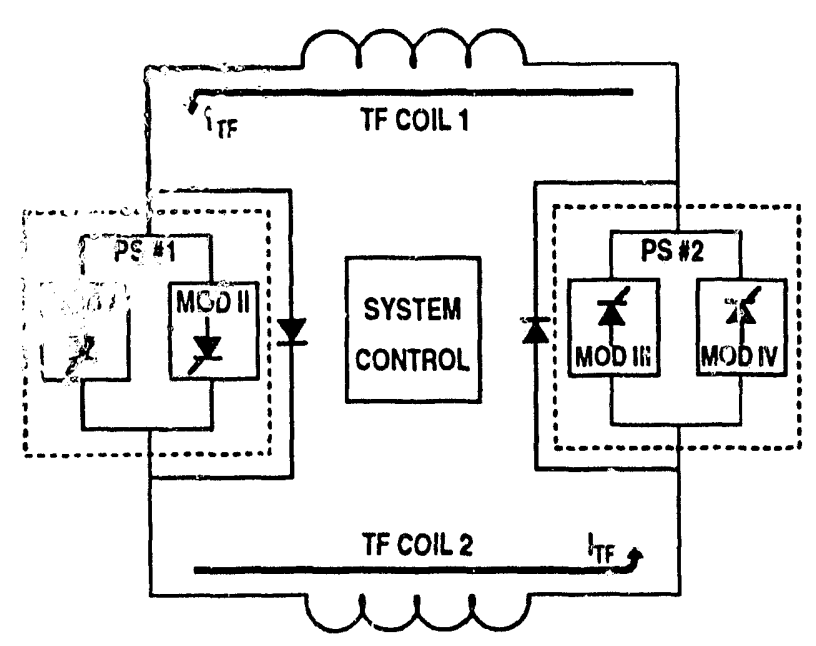

Fig. 1. TF power system configuration. 
the others in order to achieve acceptahle current and voltage sharing between parallel and series modules. The TF power supply has always operated in the series-parallel configuration, and an analysis of future operation scenarios showed no foresecable exceptions, allowing the removal of the independent loops in each module.

\section{DESIGN OBJECTIVES}

The initial objectives of the ungrade were improved current regulation and sharing, increased reliability, ease of troubleshooting, and a reduction in control loop calibration time. This required that only the voltage (E) and current (I) control loops of the power supply would be remodeled with the addition of a Programmable Logic Controller (PLC.) for some supervisory control. Further investigation revealed a need for improved fault processing capabilities and additional control from the main system console such as remote reset and breaker operation and improved fault reporting. In addition, an increase in the pulse rating of the supply was desired in preparation for future extended pulse operation. While designing a plan for integrating these changes into the existing system, it became clear that the best approach was to completely remove all of the existing control equipment. With the exception of the fault sensors, all of the fault logic circuits were to be replaced as well. The reasons for this drastic action were twofold: (1) the control boards, relays, switches, etc., were in poor condition due to operation in a less than ideal environment, including vibration, humidity and lack of filtered ventilation; and (2) the existing docusentation for the controls was lacking in credibility. Incorporating new equipment into this system just would not have corrected all of the problems associated with the aging controller components.

\section{NEW APPROACH}

The heart of the redesigned system is a new GE-Fanuc Model 90-70 PLC. The 90-70 is a VME-based series that communicates over a rack mounted back plane by way of the VME C.1 Standard fonnat. The Model 782 CPU uses a 32 bit, $16 \mathrm{MHz} 80386 \mathrm{DX}$ floating point microprocessor that executes logic at the rate of $0.4 \mu \mathrm{sec}$ per Boolean function. This PLC replaced more than 600 mechanical relays and all of the interconnections between them that previously provided control and interlock functions. The individual fault sensors monitoring the power supply, and overall system interlocks, are connected directly to the input modules of the PLC. In addition, ail digital control signals are connected directly from push buttons on the front panel or through control interface cards that buffer signals from the main control room. Sequencing and fault checking are handled by the software in the PLC. Adequate voltage isolation between the fault sensors from the individual modules is provided by opto-isolators that are integral to the front end of all inputs, up to $1500 \mathrm{~V}$. Outputs for proper control, protection and fault indication are opto-isolated drivers. The PLC is located in the systems cabinet (Fig. 2) where it is free of the vibrations that are inherent in the module control cabinets. The solid state architecture of the PLC allows it to operate in a very dirty environment without fear of moving contacts corroding due to moisture and airborne contaminants.

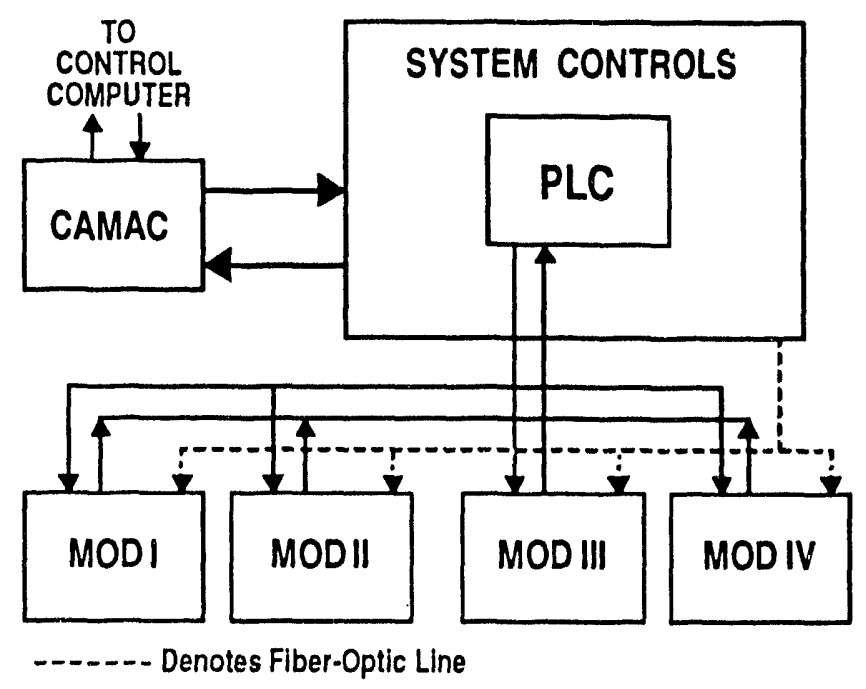

Fig. 2. TF power system control interconnect.

\section{SINGLE LOOP CONTROL}

A new control scheme utilizing an outer voltage loop and an inner current loop was developed. This replaced the five $\mathrm{E}$ and I loops that were part of the original power supply. A single GA custom-designed VME control card handles all calculations for the loop error voltage that is fed to the phase lock loop. The twelve pulses developed are fed into the VME back plane where four 16-channel fiber optic transmitter cards distribute the gate pulses to the indiviolial modules. These light driven signals insure that the modules receive their gate signals within a few nanoseconds of each other, improving the sharing of current between parallel modules. The fiber optic links also provide the necessary isolation between the high voltage converters and the control cabinet. This single loop approach accomplished several of the design objectives. The first was that current regulation and sharing were greatly enhanced. The second was a reduction in control loop maintenance time. Instead of complicated procedures to balance four loops against each other, there is a simple rrocedure for adjusting the single $\mathrm{E}$ and I loop. Another advantage was an increase in reliability by a reduction in the number of components and control loops that could possibly lead to an interruption of power supply operation.

\section{SOFTWARE CONTROL}

The PLC software has introduced flexibility to the TF supply that was unattainable with the original control system. 


\section{Fault Processing}

In the past, if any type of fault was detected in the power supply, regardless of whether it was at the system or module level, an immediate shutdown of the entire TF supply was initiated. The problem with this type of response is that the plasma shot is terminated early because the toroidal magnetic field decays, resulting in loss of critical experimental data. The PLC is able to analyze the fault, determine if it is at the system or module level, and then evaluate the need of the equipment affected (Fig. 3). Different actions will be taken by the PLC. depending on when the fault occurs, the value of the current that is being delivered, which Motor-Generator (MG) is supplying the $\mathrm{AC}$ power, and what piece of equipment has failed. Typically, any module failure after the TF has reached flattop can be handled with minimal impact on the system. The PS, which contains the faulted module, will be shutdown and the current will transfer to the free-wheeling diodes. This type of response allows the plasma shot to continue unaffected while notifying the control room that there is a problem. In most cases, the offending piece of equipment can be taken care of in less than 15 min after notification of power systems' personnel.

\section{Extended Capabilities}

The ability of the PLC to enable and inhibit the different modules has doubled the available flattop energy that can be

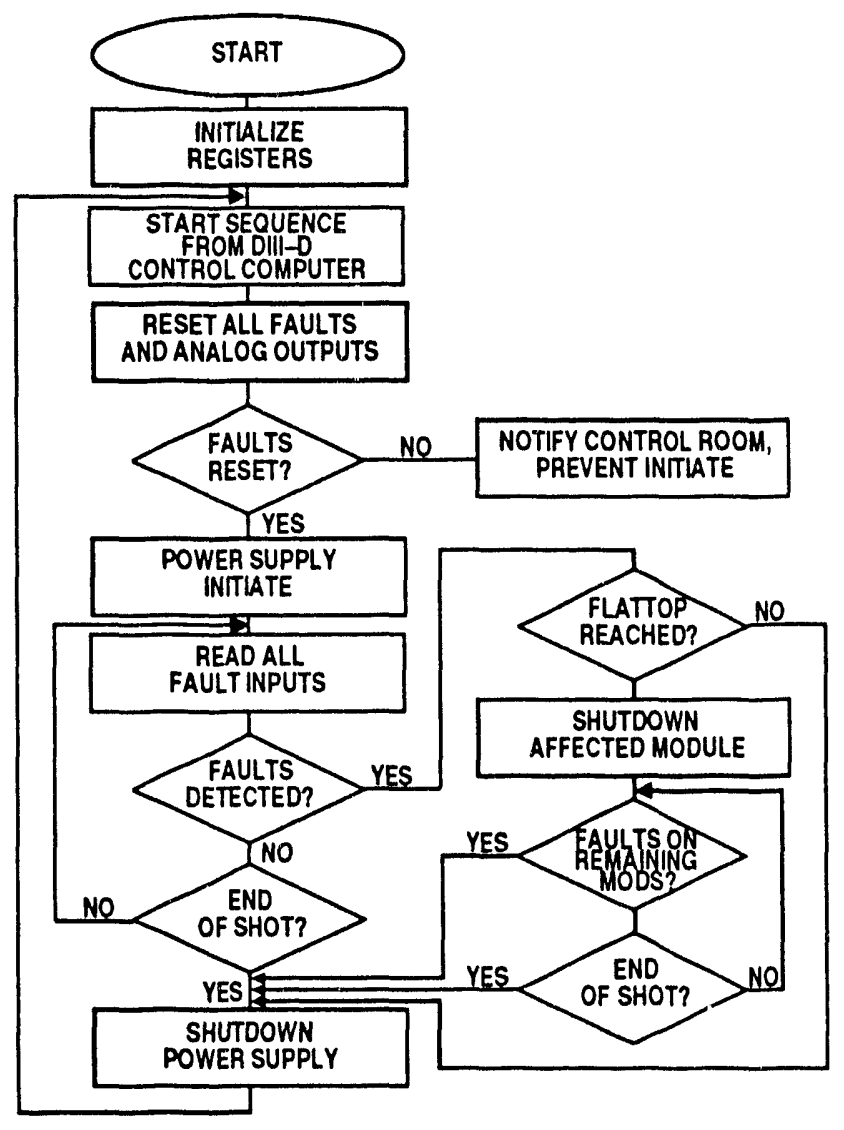

Fig. 3. PLC fault processing flowchart. delivered by the TF supply to the TF coil. This increase is realized by alternately gate blocking each of the power supplies once flattop has been reached. The series configuration of the TF supply is required only during the ramping of the toroidal field. When the two supplies are in series, a higher driving voltage is present, thereby decreasing the ramp time for the TF coil current $\left(\mathrm{I}_{\mathrm{TF}}\right)$. During flattop, a single PS can maintain the TF coil current. As with a fault, gate blocking one of the supplies transfers current to the freewheeling diodes that are connected across it. Fig. 4 shows the results of the enable-inhibit sequence and how the current transfer has negligible effect on the TF coil current. Both PS\#1 and PS\#2 are enabled and supplying current initially, until $\mathrm{I}_{\mathrm{TF}}$ reaches a predetermined programmed flattop. At this time, PS\#2 is inhibited, causing current to transfer to the diodes that are in parallel with it. PS\#1 is now supplying all the energy needs of the TF coil. When the $I^{2} t$ rating for PS\# 1 is approached, PS\#2 is enabled and brought back on line. For a short period, until PS\#2 has stabilized, both supplies are enabled. Shortly thereafter, the gates of PS\#1 are blocked, transferring the current to its free-wheeling diodes. PS\#2 now supplies the necessary energy for the TF coil until the end of the shot. In addition to the substantial increase in operational capacity, there are other benefits to running in this manner. One is the improved power factor that is drawn by the converters when they are phased on as much as possible. This occurs when one PS provides double the voltage it would nonnally supply if running in the series configuration. This
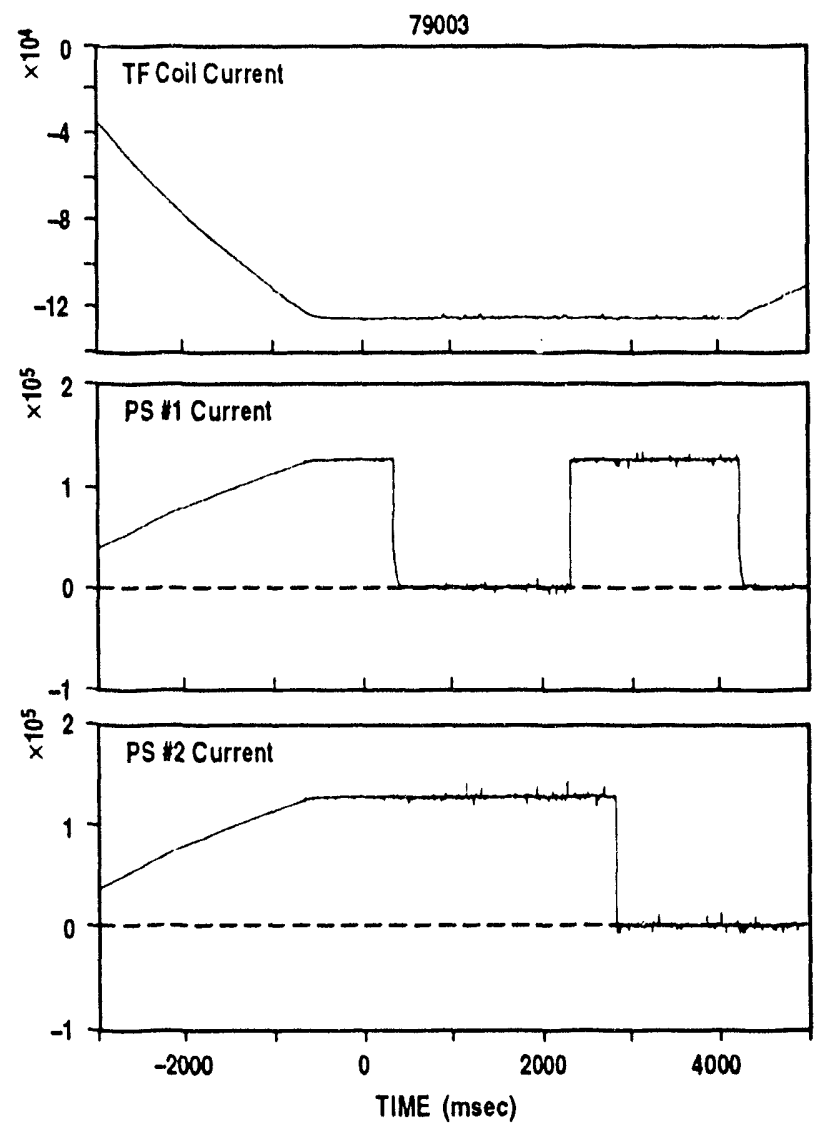

Fig. 4. TF power supply current transfer. 
results in less current draw from the MG, conserving an appreciable amount of its stored energy. The second benefit is increased life of the equipment. The present requirements of the TF coil can be supplied by running either of the PSs after flattop. The enable-inhibit sequence can run both of the PSs for one-half of the time. This shared utilization doubles the remaining lifetime of the modules.

\section{SUMMARY}

The upgrade of the control system for the TF power supply has satisfied all of the items that were set forth in the design objectives. The redesigned control loop has eliminated many hours of required calibration activities and possible lost operating time. Current sharing problems between modules have been eliminated. In addition, the new documentation and improved fault processing capabilities provided by the PLC have made troubleshooting problems a simpler process. The ability to analyze faults as they occur and take appropriate action with no affect on the plasma shot was impossible with the original control system. The enable-inhibit sequence of operation has increased the TF power supply's deliverable energy without expensive power circuit upgrade and major installation costs. 
